OPEN ACCESS

Check for updates

FAST TRACK

\title{
Effectiveness of BNT162b2 and mRNA-1273 covid-19 vaccines against symptomatic SARS-CoV-2 infection and severe covid-19 outcomes in Ontario, Canada: test negative design study
}

\author{
Hannah Chung, ${ }^{1}$ Siyi He, ${ }^{1}$ Sharifa Nasreen, ${ }^{1}$ Maria E Sundaram, ${ }^{1,2}$ Sarah A Buchan, ${ }^{1,2,3,4}$ \\ Sarah E Wilson, ${ }^{1,2,3,4}$ Branson Chen, ${ }^{1}$ Andrew Calzavara, ${ }^{1}$ Deshayne B Fell, ${ }^{1,5,6}$ \\ Peter C Austin, ${ }^{1,7}$ Kumanan Wilson, ${ }^{5,8,9}$ Kevin L Schwartz, ${ }^{1,2,3}$ Kevin A Brown, ${ }^{1,2,3}$ \\ Jonathan B Gubbay, ${ }^{3,10}$ Nicole E Basta, ${ }^{11}$ Salaheddin M Mahmud, ${ }^{12}$ Christiaan H Righolt, ${ }^{12}$ \\ Lawrence W Svenson, ${ }^{13,14,15,16}$ Shannon E MacDonald, ${ }^{15,17}$ Naveed Z Janjua, ${ }^{18,19}$ \\ Mina Tadrous, ${ }^{1,20,21}$ Jeffrey C Kwong, ${ }^{1,2,3,4,22,23}$ on behalf of the Canadian Immunization \\ Research Network (CIRN) Provincial Collaborative Network (PCN) Investigators
}

For numbered affiliations see end of the article

Correspondence to: J Kwong jeff.kwong@utoronto.ca

(or @DrjeffKwong on Twitter ORCID 0000-0002-7820-2046)

Additional material is published online only. To view please visit the journal online.

Cite this as: $B M / 2021 ; 374: n 1943$ http://dx.doi.org/10.1136/bmj.n1943

Accepted: 2 August 2021

\section{ABSTRACT}

OBJECTIVE

To estimate the effectiveness of mRNA covid-19 vaccines against symptomatic infection and severe outcomes (hospital admission or death).

DESIGN

Test negative design study.

SETTING

Ontario, Canada between 14 December 2020 and 19 April 2021.

\section{PARTICIPANTS}

324033 community dwelling people aged $\geq 16$ years who had symptoms of covid-19 and were tested for SARS-CoV-2.

INTERVENTIONS

BNT162b2 (Pfizer-BioNTech) or mRNA-1273 (Moderna) vaccine.

\section{MAIN OUTCOME MEASURES}

Laboratory confirmed SARS-CoV-2 by reverse transcription polymerase chain reaction (RT-PCR) and hospital admissions and deaths associated with SARS-CoV-2 infection. Multivariable logistic regression was adjusted for personal and clinical characteristics associated with SARS-CoV-2 and vaccine receipt to estimate vaccine effectiveness against symptomatic infection and severe outcomes.

RESULTS

Of 324033 people with symptoms, 53270 (16.4\%)

were positive for SARS-CoV-2 and 21272 (6.6\%)

\section{WHAT IS ALREADY KNOWN ON THIS TOPIC}

Clinical trials and real world effectiveness studies have shown that mRNA covid-19 vaccines are protective against symptomatic SARS-CoV-2 infections and associated severe outcomes such as hospital admission and death

Greater protection is conferred after two doses administered at least three weeks apart

\section{WHAT THIS STUDY ADDS}

Protection against symptomatic infection and severe outcomes increases gradually after one dose of an mRNA covid-19 vaccine

Although lower vaccine effectiveness was observed in older adults shortly after one dose, levels of protection similar to those of younger people were observed after longer intervals

These findings support delaying second doses when vaccine supply is limited received at least one dose of vaccine. Among participants who tested positive, 2479 (4.7\%) were admitted to hospital or died. Vaccine effectiveness against symptomatic infection observed $\geq 14$ days after one dose was $60 \%$ (95\% confidence interval $57 \%$ to $64 \%$ ), increasing from $48 \%$ ( $41 \%$ to $54 \%$ ) at $14-20$ days after one dose to $71 \%(63 \%$ to $78 \%)$ at 35-41 days. Vaccine effectiveness observed $\geq 7$ days after two doses was $91 \%$ (89\% to $93 \%$ ). Vaccine effectiveness against hospital admission or death observed $\geq 14$ days after one dose was $70 \%$ (60\% to $77 \%$ ), increasing from $62 \%$ (44\% to $75 \%$ ) at $14-20$ days to $91 \%$ ( $73 \%$ to $97 \%$ ) at $\geq 35$ days, whereas vaccine effectiveness observed $\geq 7$ days after two doses was $98 \%$ ( $88 \%$ to $100 \%$ ). For adults aged $\geq 70$ years, vaccine effectiveness estimates were observed to be lower for intervals shortly after one dose but were comparable to those for younger people for all intervals after 28 days. After two doses, high vaccine effectiveness was observed against variants with the E484K mutation.

\section{CONCLUSIONS}

Two doses of mRNA covid-19 vaccines were observed to be highly effective against symptomatic infection and severe outcomes. Vaccine effectiveness of one dose was observed to be lower, particularly for older adults shortly after the first dose.

\section{Introduction}

Understanding how efficacy estimates from clinical trials of covid-19 vaccines translate into effectiveness estimates in the real world is crucial, given differences in populations, dosing intervals, and emerging variants. ${ }^{1}$ Because of constraints on the supply of covid-19 vaccines, Canada's National Advisory Committee on Immunization recommended extending the interval between doses to a maximum of 16 weeks. ${ }^{2}$ With constraints on vaccine supply globally, determining the effectiveness of these vaccines after a single dose versus two doses is important for informing policy for many countries. ${ }^{1}$

We applied the test negative design to linked, population based health databases in Ontario, Canada (population 15 million) to evaluate vaccine effectiveness against symptomatic SARS-CoV-2 infection and severe outcomes (hospital admission or 
death) associated with SARS-CoV-2 infection for two mRNA vaccines: BNT162b2 (Pfizer-BioNTech) and mRNA-1273 (Moderna).

\section{Methods}

Study population, setting, and design

We conducted a test negative design study among community dwelling Ontarians who had symptoms consistent with covid-19. The test negative design is comparable to a nested case-control design, with individuals who have symptoms and are tested for the presence of a pathogen of interest serving as the nesting cohort. ${ }^{134}$ All Ontarians aged $\geq 16$ years, eligible for provincial health insurance, not living in long term care, and who were tested for SARS-CoV-2 between 14 December 2020 and 19 April 2021 were eligible for inclusion. We excluded those who tested positive for SARS-CoV-2 before 14 December 2020 and recipients of the ChAdOx1 (Oxford-AstraZeneca) vaccine. We restricted the analysis to individuals who had at least one relevant covid-19 symptom (based on self-report or observation, such as measured temperature), at the time of testing, which was collected on the SARS-CoV-2 test requisition form (see supplementary methods).

\section{Data sources and definitions}

Using unique encoded identifiers, we linked data from provincial SARS-CoV-2 laboratory testing, covid-19 vaccination, and health administrative datasets and analysed them at ICES (formerly the Institute for Clinical Evaluative Sciences).

\section{Outcomes}

Our first primary outcome was symptomatic SARSCoV-2 infection, ascertained by real time reverse transcription polymerase chain reaction (RT-PCR) tests on respiratory specimens, including samples from the nasopharynx (most common), nose, throat, saliva, and turbinates. ${ }^{5}$ Using data from the Ontario Laboratories Information System, which captured $91.8 \%(n=258207)$ of all provincially reported cases of laboratory confirmed covid-19 $(n=281261)$ during the study period, people who tested positive were considered as cases and those who tested negative were considered as controls. Since the dates for symptom onset were inconsistently reported in the Ontario Laboratories Information System, we used the date of specimen collection as the index date. For cases with multiple positive test results, we used the date of the first positive test result. For controls with multiple negative test results, we used the date of a randomly selected negative test result as the index date.

We obtained information on variants and mutations from the Public Health Case and Contact Management system, which contains information on the clinical course of cases and the results of screening tests for N501Y and E484K mutations and whole genome sequencing results that identify specific variant of concern lineages (alpha (B.1.1.7), beta (B.1.351), gamma (P.1)). All RT-PCR positive specimens with cycle threshold values $\leq 35$ were tested for the N501Y mutation (starting 3 February 2021) and the E484K mutation (starting 22 March 2021). ${ }^{6}$ We considered samples with positive N501Y and negative E484K mutations as alpha variants, and samples with positive $\mathrm{N} 501 \mathrm{Y}$ and E484K mutations as beta or gamma variants. We combined the latter two lineages for our analysis because there were small numbers of cases identified using whole genome sequencing.

Our second primary outcome was severe disease associated with symptomatic SARS-CoV-2 infection, defined as either hospital admission or death with a recent positive test result, using the earliest of the specimen collection date or the hospital admission or death date as the index date. We identified these outcomes using the Case and Contact Management system (for both hospital admissions and deaths), the Canadian Institute for Health Information's Discharge Abstract Database (for hospital admissions), and the Ontario Registered Persons Database (for deaths). For hospital admissions identified using the Discharge Abstract Database, a positive test result must have occurred within 14 days before or three days after admission. For deaths identified using the Registered Persons Database, a positive test result must have occurred within 30 days before death or within seven days post mortem. We used the same control group as for the first primary outcome analysis (ie, individuals with symptoms who tested negative for SARS-CoV-2).

\section{Vaccination against covid-19}

BNT162b2 became available in Ontario on 14 December 2020 and mRNA-1273 on 28 December $2020 .^{7}$ The initial vaccination phase prioritised high risk populations such as older adults living in communal settings, healthcare workers (including non-patient facing staff working in healthcare institutions), adults living in Indigenous communities, and adults aged $\geq 80$ years. $^{7}$ Ontario had initially followed the manufacturers' recommended dosing schedules (ie, a 21 day interval for BNT162b2 and a 28 day interval for mRNA-1273), but because of disruptions to vaccine supply in late January 2021, the interval was extended to 35-42 days for everyone except older adults living in communal settings, and Indigenous people. In early March, Ontario adopted the National Advisory Committee on Immunization's recommendation to delay administering the second dose by up to 16 weeks for most individuals. ${ }^{8} 9$ Eligibility expanded over time, taking into account both age (ie, graduated expansion by decreasing age) and other high risk populations, such as people with certain health conditions and their care givers, certain essential frontline workers, and those aged $\geq 18$ years living or working in communities with a high incidence of covid-19 (ie, those disproportionately affected by covid-19 and where transmission was still high). Adherence to these eligibility criteria varied across regions. As of 19 April 2021, 28\% of adults in Ontario had received at least one dose of a covid-19 vaccine. ${ }^{10}$ Comprehensive documentation of all covid-19 vaccination events in Ontario, including product, date 
of being administered, and dose number, is recorded in real time into COVaxON, a centralised covid-19 vaccine information system. We used the COVaxON file containing events up to 25 April 2021 for these analyses, which likely had records of all vaccinations delivered by 19 April 2021.

\section{Covariates}

We obtained age, sex, and postal code of residence as of 14 December 2020 from the Registered Persons Database. We obtained the number of RT-PCR tests for each participant during the three months before 14 December 2020 from the Ontario Laboratories Information System to use as a proxy for highly tested individuals at increased risk of exposure to SARS$\mathrm{CoV}-2$ infection (eg, healthcare workers and care givers of long term care residents, who must also undergo serial SARS-CoV-2 testing). To capture temporal changes in viral activity and regional vaccine roll-out, we grouped dates of testing into two week periods. We determined the presence of comorbidities that increase the risk of severe covid-19, ${ }^{11}$ identified from various databases using validated algorithms and commonly accepted diagnostic codes, which have been described elsewhere. ${ }^{12}$ Receipt of influenza vaccination (a proxy for health behaviours) was ascertained during the $2019 / 20$ or 2020/21 influenza season, or both, using physician and pharmacist billing claims in the Ontario Health Insurance Plan and Ontario Drug Benefit databases, respectively. We determined the public health unit of residence using the postal code and Statistics Canada Postal Code Conversion File Plus (version 7B) and grouped the units into larger regions. From 2016 census data we obtained information at the ecological level of dissemination area on four important social determinants of health (median household income, proportion of the working population employed as non-health essential workers (those unable to work from home), average number of people in each dwelling, and proportion of the population who self-identified as a visible minority). ${ }^{13}$ Dissemination areas generally contain 400-700 people. Supplementary eTable 1 provides details of these covariates.

\section{Statistical analysis}

To compare characteristics between test positive cases and test negative controls and between vaccinated and unvaccinated individuals, we conducted descriptive analyses and calculated standardised differences.

We used multivariable logistic regression models to estimate the odds ratio, comparing the odds of vaccination between test positive cases and test negative controls (with unvaccinated people as reference group). We estimated unadjusted and adjusted odds ratios accounting for all listed covariates. These covariates were selected a priori based on their known associations with SARS-CoV-2 infection or severity and receipt of a covid-19 vaccine v $^{1114}$ and were assessed as potential confounders (supplementary eTable 2). ${ }^{15}$ Vaccine effectiveness was calculated using the formula: vaccine effectiveness $=(1-$ odds ratio $) \times 100 \%$. We assumed those without information on exposures, outcomes, or covariates in ICES' data holdings to not have the exposure, outcome, or covariate, and they were categorised as such within the analyses.

For the primary analysis, we estimated overall vaccine effectiveness (for both mRNA vaccines combined) for those who received only one dose by their index date and those who received two doses by their index date. We considered index dates within varying intervals after vaccination.

Vaccine effectiveness was also estimated $\geq 14$ days after the first dose (among those who only received one dose) and $\geq 7$ days after the second dose, ${ }^{16}$ stratified by vaccine product (BNT162b2 or mRNA-1273), age group (16-39, 40-69, and $\geq 70$ years), sex, presence of any comorbidity, epidemic wave (index dates 14 December 2020 to 7 February 2021, representing wave 2 in Ontario; 8 February 2021 to 21 March 2021, representing the period between wave 2 and wave 3; and 22 March 2021 to 19 April 2021, representing wave 3), and variant (earlier variant versus alpha versus beta or gamma). We also estimated vaccine effectiveness by varying intervals after vaccination, stratified by age group.

We repeated these analyses for severe outcomes, with adjustments to the intervals after vaccination due to reduced sample sizes. For example, we evaluated vaccine effectiveness for the entire period ( $\geq 0$ days) after receipt of the second dose.

Finally, to assess whether systematic differences between vaccinated and unvaccinated individuals were adequately controlled for in the main analyses, in a sensitivity analysis we assessed vaccine effectiveness against symptomatic infection and severe outcomes by varying intervals among only those who were vaccinated (treating people vaccinated 0-13 days before the test as reference group). ${ }^{17}$

All analyses were conducted using SAS version 9.4 (SAS Institute, Cary, NC). Tests were two sided, with $\mathrm{P}<0.05$ considered as significant.

\section{Patient and public involvement}

Although study participants contributed in important ways to this research, it was not feasible to involve them in the design, conduct, reporting, or dissemination plans of our research. We did not involve members of the public in this research owing to resource and time constraints.

\section{Results}

Overall, 2171449 unique individuals were tested for SARS-CoV-2 from 14 December 2020 to 19 April 2021. After excluding those who had SARS-CoV-2 infection before the study period and those who had received the ChAdOx1 vaccine, $60.5 \%$ of those remaining did not have symptoms consistent with covid-19 or had no information on symptoms recorded in the Ontario Laboratories Information System, 24.4\% were recorded as asymptomatic, and $15.1 \%$ had symptoms consistent with covid-19 recorded at the time of 
testing (fig 1). Grouped together, those with covid-19like symptoms and those deemed asymptomatic had similar characteristics to the remaining individuals, except for covid-19 vaccine uptake, public health unit region, and number of previous SARS-CoV-2 tests (supplementary eTable 3).

Of the 324033 people with symptoms who were tested, 53270 (16.4\%) tested positive for SARS-CoV-2, 42567 (79.9\%) had information available on tests for variants, $21272(6.6 \%)$ had received at least one dose of mRNA vaccine, and 4894 (1.5\%) had received two doses (table 1). Among test positive cases, 2479 (4.7\%) had a severe outcome, of whom 2035 were admitted to hospital and 444 died. Most cases admitted to hospital tested positive before or on the admission date (1728, $84.9 \%$ ) and nearly all tested positive before death. Test positive cases were more likely to be male, to reside in Peel region or Toronto, and to have had no SARS-CoV-2 tests during the three months before the vaccination programme, less likely to have received an influenza vaccine, and more likely to reside in neighbourhoods with lower income, more people in each dwelling, and greater proportions of essential workers and people from visible minorities (table 1). Vaccinated people were older, less likely to be male, and more likely to have had multiple SARS-CoV-2 tests during the three months before the vaccination programme, a comorbidity, and received an influenza vaccine. Compared with recipients of the mRNA-1273 vaccine, recipients of the BNT162b2 vaccine were younger, more likely to be female, and less likely to have a comorbidity (supplementary eTable 4). The distribution of these vaccine products also differed by public health unit regions. Most participants (77\% for BNT162b2, 76\% for mRNA-1273) had received only one dose by the index date. Supplementary eFigure 1 presents the distribution of vaccine product over the study period.

Against symptomatic infection, adjusted vaccine effectiveness observed $\geq 14$ days after only one dose was $60 \%$ (95\% confidence interval $57 \%$ to $64 \%$ ). This increased from $48 \%$ (41\% to $54 \%$ ) at $14-20$ days to a plateau of $71 \%(63 \%$ to $78 \%$ ) at $35-41$ days (fig 2, supplementary eTable 5). A $16 \%$ increase in risk of symptomatic infection was observed 7-13 days after one dose (vaccine effectiveness $-16 \%,-26 \%$ to $-6 \%$ ), but no increase was observed 0-6 days after one dose. Vaccine effectiveness observed $\geq 7$ days after two doses was $91 \%$ (89\% to $93 \%)$. Against severe outcomes of hospital admission or death, vaccine effectiveness observed $\geq 14$ days after one dose was $70 \%$ (60\% to $77 \%$ ), increasing from $62 \%$ (44\% to $75 \%)$ at $14-20$ days to $91 \%$ ( $73 \%$ to $97 \%$ ) at $\geq 35$ days, whereas vaccine effectiveness observed $\geq 7$ days after two doses was $98 \%$ (88\% to $100 \%$ ) (fig 3, supplementary eTable 5).

In subgroup analyses of vaccine effectiveness against symptomatic infection, higher effectiveness was observed $\geq 14$ days after only one dose of mRNA-1273 versus one dose of BNT162b2 (which was consistent across all age groups), for younger people versus adults aged $\geq 70$ years, for those with no comorbidities versus those with comorbidities, and against the earlier variant and alpha variant versus beta or gamma variant (although 95\% confidence intervals for vaccine effectiveness estimates for variants overlapped) (fig 4, supplementary eTable 6). Vaccine effectiveness

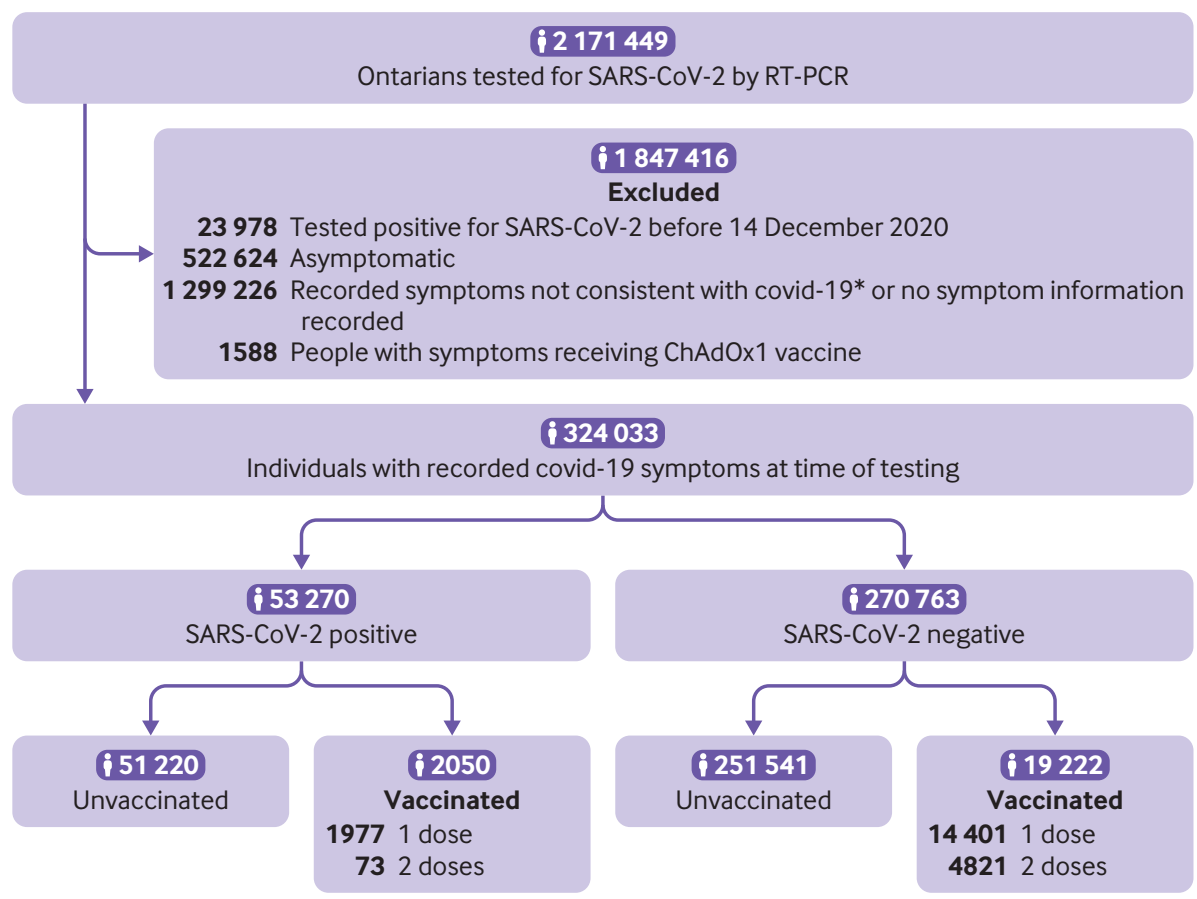

Fig 1 | Community dwelling people aged $\geq 16$ years included in tested cohort between 14 December 2020 and 19 April 2021 in Ontario, Canada. *Recorded symptoms not consistent with covid-19 (eg, anxiety, cancer, falls). RT$\mathrm{PCR}=$ reverse transcription polymerase chain reaction; ChAd0x1=0xford-AstraZeneca vaccine 
Table 1 | Characteristics of participants with symptoms tested for SARS-CoV-2 between 14 December 2020 and 19 April 2021 in Ontario, Canada. Values are numbers (percentages) unless stated otherwise

\begin{tabular}{|c|c|c|c|c|c|c|}
\hline \multirow[b]{2}{*}{ Characteristics } & \multicolumn{2}{|c|}{ SARS-CoV-2 status } & \multirow[b]{2}{*}{$\begin{array}{l}\text { Standardised } \\
\text { difference* }\end{array}$} & \multicolumn{2}{|c|}{ Vaccination status } & \multirow[b]{2}{*}{$\begin{array}{l}\text { Standardised } \\
\text { difference }{ }^{\star}\end{array}$} \\
\hline & $\begin{array}{l}\text { Positive } \\
(n=53270)\end{array}$ & $\begin{array}{l}\text { Negative } \\
(n=270763)\end{array}$ & & $\begin{array}{l}\geq 1 \text { dose } \\
\text { mRNA vaccine } \\
(n=21272)\end{array}$ & $\begin{array}{l}\text { Unvaccinated } \\
(n=302761)\end{array}$ & \\
\hline \multicolumn{7}{|l|}{ Vaccine dose: } \\
\hline At least 1 & $2050(3.8)$ & $19222(7.1)$ & - & - & - & - \\
\hline 2 & $73(0.1)$ & $4821(1.8)$ & - & - & - & - \\
\hline Positivity to SARS-CoV-2 type: & - & - & - & $2050(9.6)$ & $51220(16.9)$ & - \\
\hline Earlier variant & - & - & - & $579(2.7)$ & $27510(9.1)$ & - \\
\hline Alpha (B.1.1.7) & - & - & - & $807(3.8)$ & $12282(4.1)$ & - \\
\hline Beta (B.1.351) or gamma (P.1) (variants with E484K mutation) & - & - & - & $98(0.5)$ & $1291(0.4)$ & - \\
\hline Mean (SD) age (years) & $42.4(17.1)$ & $43.2(17.8)$ & 0.04 & $51.8(20.8)$ & $42.4(17.3)$ & 0.49 \\
\hline \multicolumn{7}{|l|}{ Age group (years): } \\
\hline $16-29$ & $15175(28.5)$ & $72238(26.7)$ & 0.04 & $3457(16.3)$ & $83956(27.7)$ & 0.28 \\
\hline $30-39$ & $10024(18.8)$ & $59326(21.9)$ & 0.08 & $4011(18.9)$ & $65339(21.6)$ & 0.07 \\
\hline $40-49$ & $9642(18.1)$ & $46225(17.1)$ & 0.03 & $3287(15.5)$ & $52580(17.4)$ & 0.05 \\
\hline $50-59$ & $9460(17.8)$ & $40874(15.1)$ & 0.07 & $3112(14.6)$ & $47222(15.6)$ & 0.03 \\
\hline $60-69$ & $5279(9.9)$ & $27342(10.1)$ & 0.01 & $2261(10.6)$ & $30360(10.0)$ & 0.02 \\
\hline $70-79$ & $2426(4.6)$ & $14888(5.5)$ & 0.04 & $2272(10.7)$ & $15042(5.0)$ & 0.21 \\
\hline$\geq 80$ & $1264(2.4)$ & $9870(3.6)$ & 0.07 & $2872(13.5)$ & $8262(2.7)$ & 0.40 \\
\hline Males & $25993(48.8)$ & $112501(41.5)$ & 0.15 & $6013(28.3)$ & $132481(43.8)$ & 0.33 \\
\hline \multicolumn{7}{|l|}{ Public health unit regiont: } \\
\hline Central East & $2624(4.9)$ & $29194(10.8)$ & 0.22 & $1969(9.3)$ & $29849(9.9)$ & 0.02 \\
\hline Central West & $8322(15.6)$ & 48419 (17.9) & 0.06 & $3853(18.1)$ & $52888(17.5)$ & 0.02 \\
\hline Durham & $1433(2.7)$ & $8583(3.2)$ & 0.03 & $522(2.5)$ & $9494(3.1)$ & 0.04 \\
\hline Eastern & $689(1.3)$ & $15147(5.6)$ & 0.24 & $1087(5.1)$ & $14749(4.9)$ & 0.01 \\
\hline North & $1753(3.3)$ & $31321(11.6)$ & 0.32 & $2251(10.6)$ & $30823(10.2)$ & 0.01 \\
\hline Ottawa & $417(0.8)$ & $3144(1.2)$ & 0.04 & $446(2.1)$ & $3115(1.0)$ & 0.09 \\
\hline Peel & $13515(25.4)$ & $32981(12.2)$ & 0.34 & 2395 (11.3) & $44101(14.6)$ & 0.10 \\
\hline South West & $7562(14.2)$ & $39316(14.5)$ & 0.01 & $3885(18.3)$ & $42993(14.2)$ & 0.11 \\
\hline Toronto & $12458(23.4)$ & $45540(16.8)$ & 0.16 & $3462(16.3)$ & $54536(18.0)$ & 0.05 \\
\hline York & $4278(8.0)$ & $15995(5.9)$ & 0.08 & $1323(6.2)$ & $18950(6.3)$ & 0.00 \\
\hline \multicolumn{7}{|l|}{ Biweekly period of test: } \\
\hline 14 Dec 2020 to 27 Dec 2020 & $4139(7.8)$ & $27456(10.1)$ & 0.08 & $13(0.1)$ & $31582(10.4)$ & 0.48 \\
\hline 28 Dec 2020 to 10 Jan 2021 & 6870 (12.9) & $26993(10.0)$ & 0.09 & $335(1.6)$ & 33528 (11.1) & 0.40 \\
\hline $11 \operatorname{Jan} 2021$ to $24 \operatorname{Jan} 2021$ & $4864(9.1)$ & $26747(9.9)$ & 0.03 & $1068(5.0)$ & $30543(10.1)$ & 0.19 \\
\hline $25 \operatorname{Jan} 2021$ to 7 Feb 2021 & $3539(6.6)$ & $24276(9.0)$ & 0.09 & $1204(5.7)$ & $26611(8.8)$ & 0.12 \\
\hline 8 Feb 2021 to 21 Feb 2021 & $3595(6.7)$ & $24800(9.2)$ & 0.09 & $1031(4.8)$ & $27364(9.0)$ & 0.17 \\
\hline 22 Feb 2021 to 7 Mar 2021 & $3539(6.6)$ & $30760(11.4)$ & 0.17 & $1491(7.0)$ & $32808(10.8)$ & 0.13 \\
\hline 8 Mar 2021 to 21 Mar 2021 & $5134(9.6)$ & $32776(12.1)$ & 0.08 & $2790(13.1)$ & $35120(11.6)$ & 0.05 \\
\hline 22 Mar 2021 to 4 Apr 2021 & $8338(15.7)$ & $35910(13.3)$ & 0.07 & $4814(22.6)$ & $39434(13.0)$ & 0.25 \\
\hline 5 Apr 2021 to 19 Apr 2021 & $13252(24.9)$ & $41045(15.2)$ & 0.24 & $8526(40.1)$ & $45771(15.1)$ & 0.58 \\
\hline \multicolumn{7}{|l|}{ No of tests in past three months: } \\
\hline 0 & $43713(82.1)$ & $189786(70.1)$ & 0.28 & $11588(54.5)$ & $221911(73.3)$ & 0.40 \\
\hline 1 & $7151(13.4)$ & $54827(20.2)$ & 0.18 & $4338(20.4)$ & $57640(19.0)$ & 0.03 \\
\hline$\geq 2$ & $2406(4.5)$ & $26150(9.7)$ & 0.20 & $5346(25.1)$ & $23210(7.7)$ & 0.49 \\
\hline Any comorbidity $\neq$ & $23212(43.6)$ & $127974(47.3)$ & 0.07 & $12218(57.4)$ & $138968(45.9)$ & 0.23 \\
\hline $2019-2020$ and/or 2020-21 influenza vaccine & $13751(25.8)$ & $89395(33.0)$ & 0.16 & $9587(45.1)$ & $93559(30.9)$ & 0.30 \\
\hline \multicolumn{7}{|l|}{ Household income fiftht§: } \\
\hline 1 (lowest) & $11878(22.3)$ & $47944(17.7)$ & 0.11 & $3750(17.6)$ & $56072(18.5)$ & 0.02 \\
\hline 2 & $11154(20.9)$ & $51470(19.0)$ & 0.05 & $4146(19.5)$ & $58478(19.3)$ & 0.00 \\
\hline 3 & $11477(21.5)$ & $52628(19.4)$ & 0.05 & $4233(19.9)$ & $59872(19.8)$ & 0.00 \\
\hline 4 & $10146(19.0)$ & $56676(20.9)$ & 0.05 & $4513(21.2)$ & $62309(20.6)$ & 0.02 \\
\hline 5 (highest) & $8359(15.7)$ & $60774(22.4)$ & 0.17 & $4540(21.3)$ & $64593(21.3)$ & 0.00 \\
\hline \multicolumn{7}{|l|}{ Essential workers fifth (\%)+ף: } \\
\hline $1(0-32.5)$ & $6440(12.1)$ & $50664(18.7)$ & 0.18 & $3917(18.4)$ & $53187(17.6)$ & 0.02 \\
\hline $2(32.5-42.3)$ & $11225(21.1)$ & $60040(22.2)$ & 0.03 & $4664(21.9)$ & $66601(22.0)$ & 0.00 \\
\hline $3(42.3-49.8)$ & $11106(20.8)$ & $56108(20.7)$ & 0.00 & $4468(21.0)$ & $62746(20.7)$ & 0.01 \\
\hline $4(50.0-57.5)$ & $11576(21.7)$ & $52849(19.5)$ & 0.05 & $4211(19.8)$ & $60214(19.9)$ & 0.00 \\
\hline $5(57.5-100)$ & $12519(23.5)$ & $49067(18.1)$ & 0.13 & $3859(18.1)$ & $57727(19.1)$ & 0.02 \\
\hline \multicolumn{7}{|l|}{ No (range) of people per dwelling fiftht: } \\
\hline $1(0-2.1)$ & $5781(10.9)$ & $51852(19.2)$ & 0.23 & $4277(20.1)$ & $53356(17.6)$ & 0.06 \\
\hline $2(2.2-2.4)$ & $6641(12.5)$ & $52326(19.3)$ & 0.19 & $4219(19.8)$ & $54748(18.1)$ & 0.04 \\
\hline $3(2.5-2.6)$ & $5633(10.6)$ & 37229 (13.7) & 0.10 & $3020(14.2)$ & 39842 (13.2) & 0.03 \\
\hline $4(2.7-3.0)$ & $12967(24.3)$ & $63774(23.6)$ & 0.02 & $4874(22.9)$ & $71867(23.7)$ & 0.02 \\
\hline $5(3.1-5.7)$ & $21833(41.0)$ & $63459(23.4)$ & 0.38 & $4709(22.1)$ & $80583(26.6)$ & 0.10 \\
\hline
\end{tabular}




\begin{tabular}{|c|c|c|c|c|c|c|}
\hline \multirow[b]{2}{*}{ Characteristics } & \multicolumn{2}{|c|}{ SARS-CoV-2 status } & \multirow[b]{2}{*}{$\begin{array}{l}\text { Standardised } \\
\text { difference* }^{\star}\end{array}$} & \multicolumn{2}{|c|}{ Vaccination status } & \multirow[b]{2}{*}{$\begin{array}{l}\text { Standardised } \\
\text { difference* }\end{array}$} \\
\hline & $\begin{array}{l}\text { Positive } \\
(n=53270)\end{array}$ & $\begin{array}{l}\text { Negative } \\
(n=270763)\end{array}$ & & $\begin{array}{l}\geq 1 \text { dose } \\
\text { mRNA vaccine } \\
(n=21272)\end{array}$ & $\begin{array}{l}\text { Unvaccinated } \\
(n=302761)\end{array}$ & \\
\hline \multicolumn{7}{|c|}{ Self-identified visible minority fifth (\%) ${ }^{* *}$ : } \\
\hline $1(0.0-2.2)$ & $4437(8.3)$ & $51919(19.2)$ & 0.32 & $4133(19.4)$ & $52223(17.2)$ & 0.06 \\
\hline $2(2.2-7.5)$ & $5752(10.8)$ & $55124(20.4)$ & 0.27 & $4592(21.6)$ & $56284(18.6)$ & 0.07 \\
\hline $3(7.5-18.7)$ & $7223(13.6)$ & $51122(18.9)$ & 0.14 & $3982(18.7)$ & $54363(18.0)$ & 0.02 \\
\hline $4(18.7-43.5)$ & $10718(20.1)$ & $53691(19.8)$ & 0.01 & $3974(18.7)$ & $60435(20.0)$ & 0.03 \\
\hline $5(43.5-100)$ & $24736(46.4)$ & $56876(21.0)$ & 0.56 & $4438(20.9)$ & $77174(25.5)$ & 0.11 \\
\hline
\end{tabular}

*Values $>0.10$ are considered clinically relevant.

tSum of counts does not equal column total because of individuals with missing information ( $1.0 \%)$ for this characteristic.

$\ddagger$ Comorbidities include chronic respiratory diseases, chronic heart diseases, hypertension, diabetes, immunocompromising conditions due to underlying diseases or treatment, autoimmune

diseases, chronic kidney disease, advanced liver disease, dementia/frailty, and history of stroke or transient ischaemic attack.

§Includes variable cut-off values in each city/census area to account for cost of living. A dissemination area (DA) being in the first fifth means it is among the lowest $20 \%$ of DAs in its city by income.

१Percentage of people in area working in sales and service occupations; trades, transport and equipment operators, and related occupations; natural resources, agriculture, and related

production occupations; and occupations in manufacturing and utilities. Census counts for people are randomly rounded up or down to the nearest number divisible by 5 , which results in some minor imprecision.

**Percentage of people in area who self-identified as a visible minority. Census counts for people are randomly rounded up or down to the nearest number divisible by 5 , which results in some minor imprecision.

estimates observed $\geq 7$ days after two doses were, however, high (all $\geq 88 \%$ ) and comparable across all subgroups, including against variants with the E484K mutation. Against severe outcomes, higher vaccine effectiveness was observed $\geq 14$ days after one dose for those aged 16-39 years, but effectiveness estimates after two doses were mostly similar across subgroups (fig 4, supplementary eTable 7).

Among adults aged $\geq 70$ years, vaccine effectiveness against symptomatic infection after one dose was observed to be $64 \%(46 \%$ to $76 \%$ ) at $28-34$ days and $85 \%$ (38\% to $97 \%$ ) at $42-48$ days, whereas comparable effectiveness estimates were achieved sooner after one dose for younger people (fig 5, supplementary eTable 8). Furthermore, vaccine effectiveness against severe outcomes was observed to be similar at $\geq 35$ days after one dose $(93 \%, 71 \%$ to $98 \%)$ as after two doses (97\%, $86 \%$ to $99 \%$ ).

In the sensitivity analysis restricted to vaccinated people with those vaccinated 0-13 days before the index date serving as the reference group, vaccine effectiveness estimates against symptomatic infection were observed to be similar to those of the main analyses (supplementary eTable 5). For hospital admissions and deaths, however, the estimates differed for earlier vaccination intervals.

\section{Discussion}

Using the test negative study design, which mitigates selection bias from differences in health seeking

$\Phi$ Received only 1 dose $\Phi$ Received 2 doses

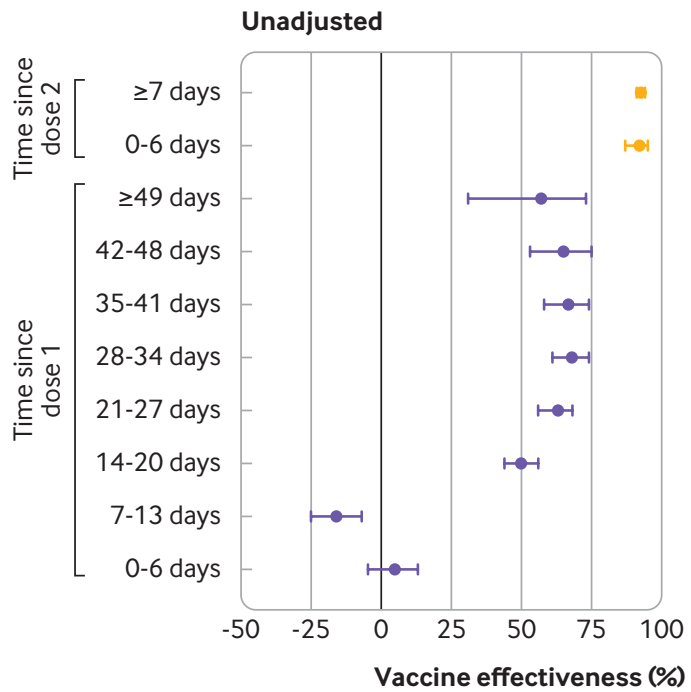

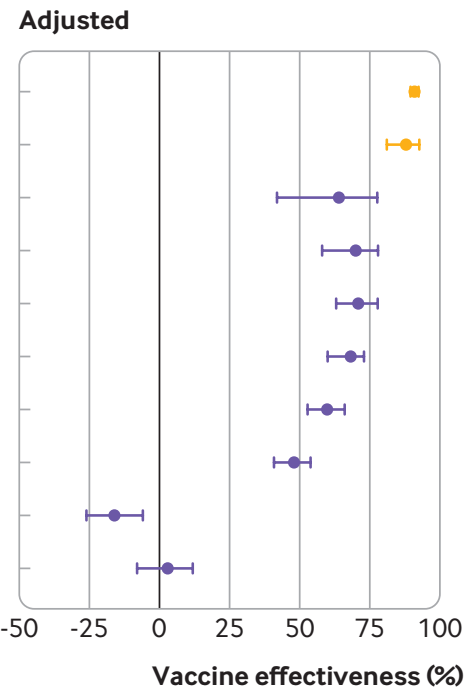

Fig 2 | Unadjusted and adjusted vaccine effectiveness estimates of covid-19 mRNA vaccines (BNT162b2, mRNA-1273) against laboratory confirmed symptomatic SARS-CoV-2 infection by various intervals, between 14 December 2020 and 19 April 2021 in Ontario, Canada. Models were adjusted for age, sex, public health unit region, biweekly period of test, number of SARS-CoV-2 tests in the three months before 14 December 2020, presence of any comorbidity increasing the risk of severe covid-19, receipt of influenza vaccination in current or previous influenza season, and fifths of neighbourhood level household income, number of people in each dwelling, proportion of people employed as non-health essential workers, and self-identified visible minority 


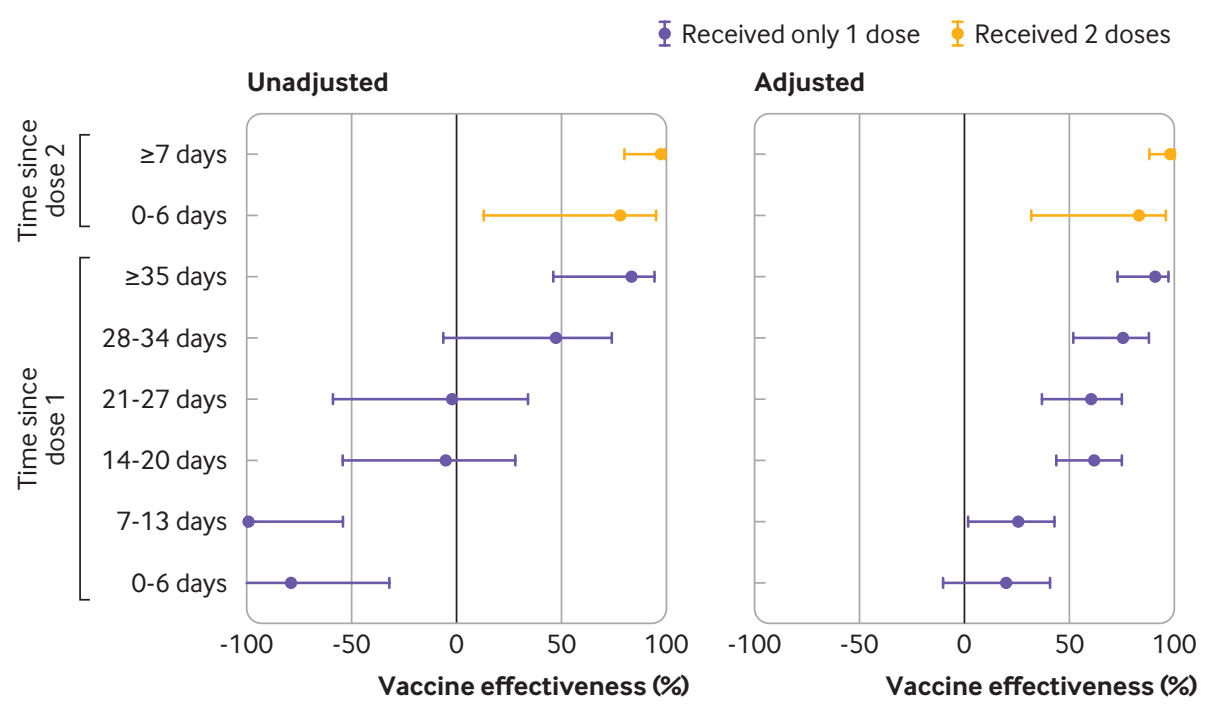

Fig 3 | Unadjusted and adjusted vaccine effectiveness estimates of covid-19 mRNA vaccines (BNT162b2, mRNA-1273) against severe outcomes (hospital admission or death) associated with laboratory confirmed symptomatic SARSCoV-2 infection by various intervals, between 14 December 2020 and 19 April 2021 in Ontario, Canada. Models were adjusted for age, sex, public health unit region, biweekly period of test, number of SARS-CoV-2 tests in the three months before 14 December 2020, presence of any comorbidity increasing the risk of severe covid-19, receipt of influenza vaccination in current or previous influenza season, and fifths of neighbourhood level household income, number of people in each dwelling, proportion of people employed as non-health essential workers, and selfidentified visible minority

behaviour between vaccinated and unvaccinated people, we estimated high $(>90 \%)$ vaccine effectiveness of mRNA vaccines BNT162b2 and mRNA1273 against symptomatic SARS-CoV-2 infection with full vaccination ( $\geq 7$ days after a second dose), and moderate (about 50-70\%) vaccine effectiveness with partial vaccination ( $\geq 14$ days after only one dose). Estimates for both full and partial vaccination were about 10 percentage points higher against hospital admission or death than against symptomatic infection. Vaccine effectiveness generally was observed to increase over time after one dose; however, we also observed a slightly increased risk of symptomatic infection on days 7-13 after one dose, compared with no vaccination. In subgroup analyses, we observed lower vaccine effectiveness against symptomatic infection in adults aged $\geq 70$ years and those with comorbidities, but a higher effectiveness after one dose of mRNA-1273 than after one dose of BNT162b2. Vaccine effectiveness was, however, observed to be consistently high across subgroups for fully vaccinated people, and also for older adults after longer intervals from a first dose.

\section{Comparison with other studies}

Our findings for fully vaccinated people are comparable with efficacy estimates from clinical trials and other real world effectiveness estimates reported in a range of settings. ${ }^{16}$ 18-31 Existing evidence for effectiveness estimates of one dose of mRNA vaccines from observational studies is heterogeneous, ${ }^{16} 27$ 30-32 with estimates for symptomatic infection ranging from $57 \%$ (95\% confidence interval $50 \%$ to $63 \%)^{16}$ to $72 \%$ $(58 \% \text { to } 86 \%)^{31}$ and post hoc calculations from efficacy trials about $90 \% .^{33} 34$ Similar heterogeneity has been found among one dose effectiveness estimates in older adults, ${ }^{253235}$ with estimates generally lower for older adults after one dose, ${ }^{16}{ }^{32}$ and increasing over time. In our analysis we observed an effectiveness against symptomatic infection of $63 \% \quad(95 \%$ confidence interval $40 \%$ to $72 \%$ ) $\geq 49$ days after only one dose, in keeping with the findings of several other studies. ${ }^{1630}$ In addition, we found that one dose of mRNA-1273 was associated with significantly higher effectiveness against symptomatic infection than one dose of BNT162b2. Differences in characteristics between recipients of the two vaccines might explain this finding, but similar results were also found in another Canadian province. ${ }^{36}$ Findings were, however, inconsistent in other studies that compared vaccine effectiveness between products after one dose; some found a trend towards higher effectiveness against infection using mRNA-1273, ${ }^{3738}$ whereas others found no difference. ${ }^{3940}$ However, the populations in each of these studies were more homogeneous than ours (eg, adults aged $\leq 40$ years, healthcare workers, veterans). Our analysis also reflects extant evidence that vaccine effectiveness against symptomatic infection ${ }^{16}$ and covid-19 associated hospital admissions ${ }^{1923}$ increases to high levels after a second dose, even in older adults. Lastly, our finding that two doses of mRNA vaccines was not associated with appreciable vaccine escape by lineage alpha or variants with the E484K mutation (beta and gamma) is notable.

In our study, we observed an increased risk of infection 7-13 days after vaccination. Other studies also found an increased risk of SARS-CoV-2 infection up to 14 days after one dose. ${ }^{25} 314142$ This could be due to an 


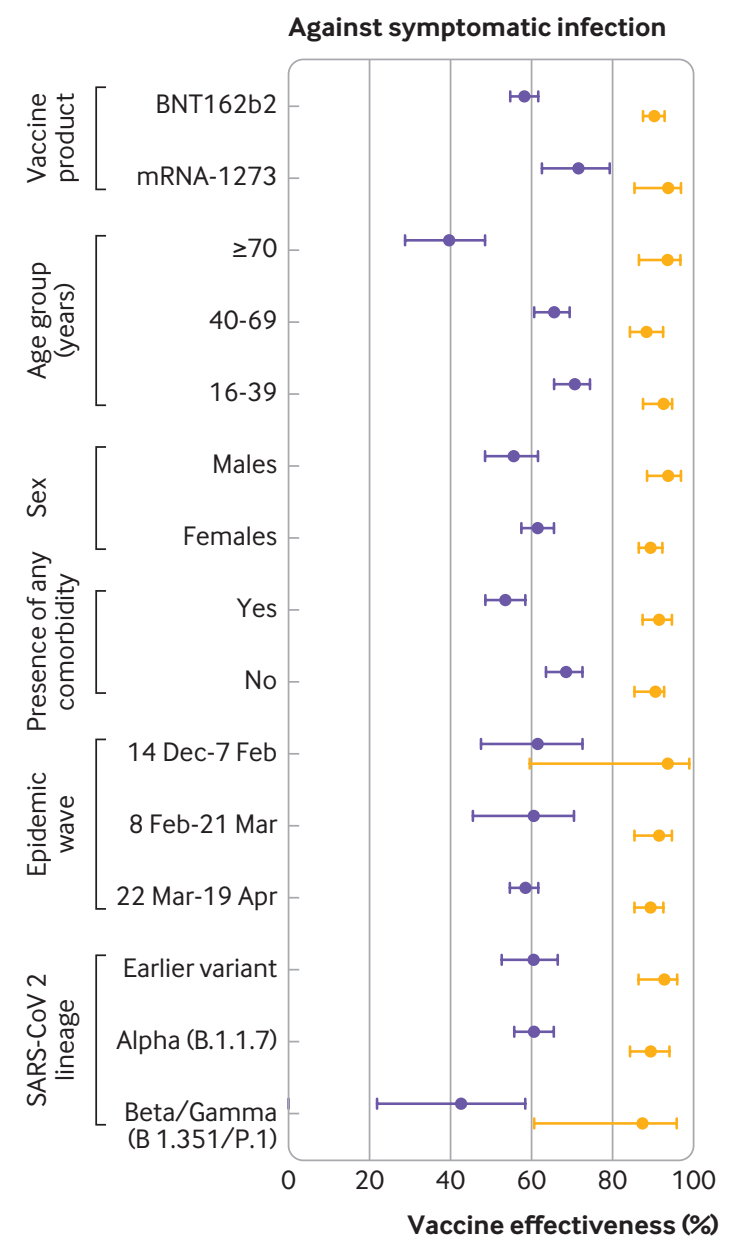

$\Phi \geq 14$ days after dose 1 After dose $2^{*}$

Against severe outcomes

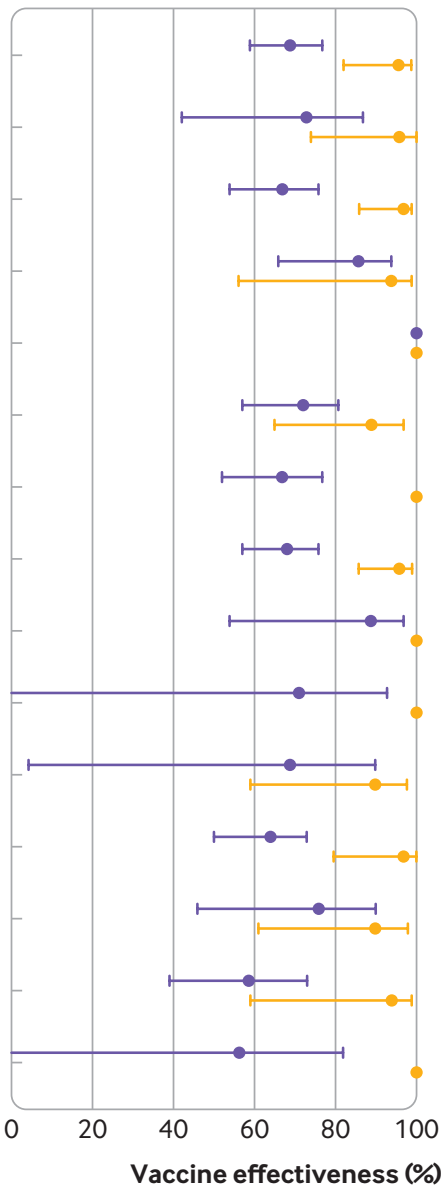

Fig 4 | Adjusted vaccine effectiveness estimates $\geq 14$ days after dose 1 (for those who received only one dose) and $\geq 0$ days after dose 2 of covid-19 mRNA vaccines (BNT162b2, mRNA-1273) by various factors, including vaccine product, patient characteristics, epidemic wave, and SARS-CoV-2 lineage against laboratory confirmed symptomatic SARSCoV-2 infection and severe outcomes (hospital admission or death) between 14 December 2020 and 19 April 2021 in Ontario, Canada. Models were adjusted for age, sex, public health unit region, biweekly period of test, number of SARS-CoV-2 tests in the three months before 14 December 2020, presence of any comorbidity increasing the risk of severe covid-19, receipt of influenza vaccination in current or previous influenza season, and fifths of neighbourhood level household income, number of people living in each dwelling, proportion of people employed as non-health essential workers, and self-identified visible minority (unless adjusted variable was used for stratification). *For vaccine effectiveness estimates against symptomatic SARS-CoV- 2 infection, this interval was $\geq 7$ days after dose 2. Against severe outcomes, vaccine effectiveness was evaluated for the entire period ( $\geq 0$ days) after receipt of the second dose owing to the small number of outcomes. For subgroup analyses by characteristic and SARS-CoV-2 lineage, individuals vaccinated with either mRNA vaccine were included

increase in exposures to SARS-CoV-2 after vaccination. Individuals might assume that they are protected against infection immediately after vaccination and engage in higher risk behaviours before a sufficient immune response has developed. Indeed, about 20\% of the US public believe that protection is conferred either immediately or 1-2 weeks after the first vaccine dose. ${ }^{43}$ Future studies should examine the potential role of behavioural changes after the first dose of covid-19 vaccines. This finding could also be due a higher baseline risk of infection among those who were initially prioritised to receive the vaccine, which might not have been adequately controlled for in our models. A vaccine effectiveness estimate of close to $0 \%$ at 0-6 days after one dose, however, provides a level of validation that we had accounted for the differences between vaccinated and unvaccinated people.

\section{Limitations of this study}

This study has some limitations. Firstly, our study sample was limited to those with symptoms of covid-19 recorded in the Ontario Laboratories Information System, which decreased our potential sample size considerably, from 2171449 people who were tested for SARS-CoV-2 to 324033 who had relevant covid-19 symptoms recorded in the information repository. Not all laboratories in Ontario currently have the information technology infrastructure to submit information on symptoms (or documentation of asymptomatic testing) recorded 
Against symptomatic infection

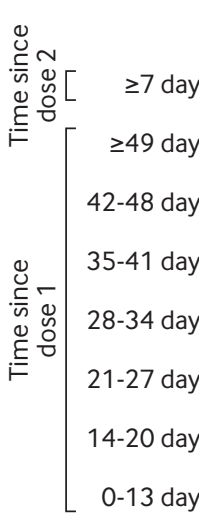

$\geq 70$ years

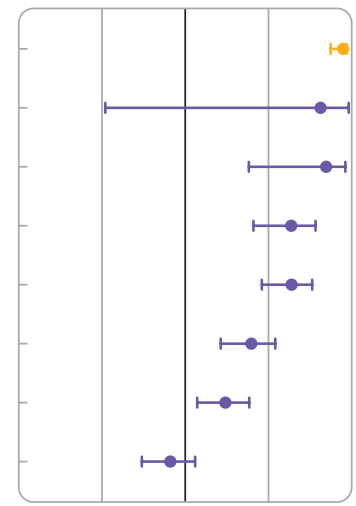

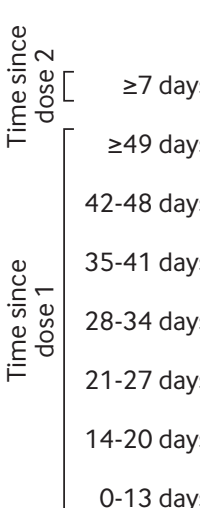

40-69 years
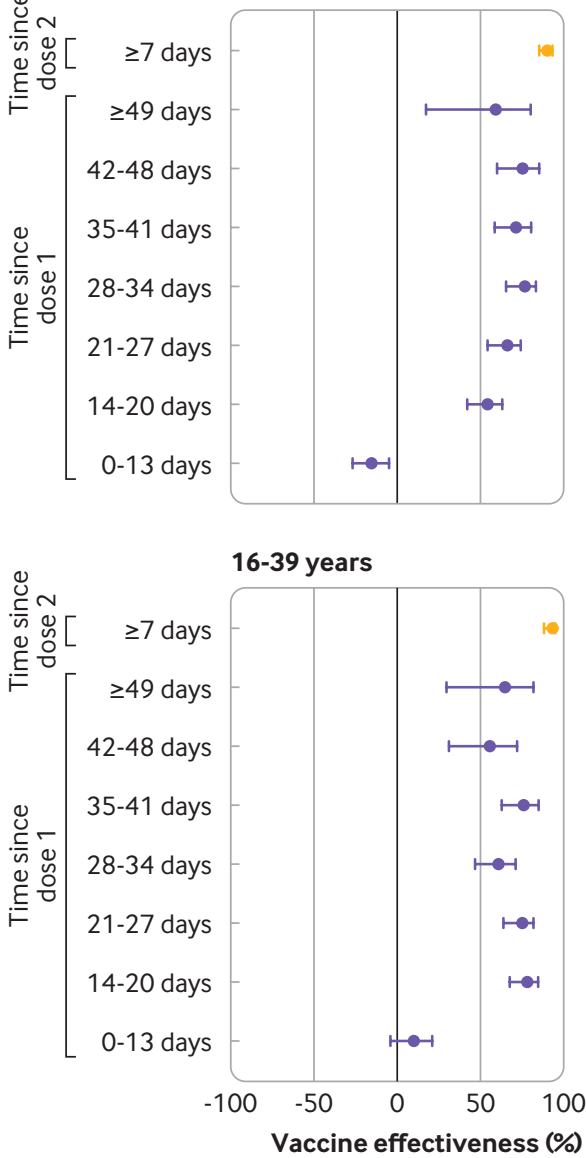

$\Phi$ Received only 1 dose $\Phi$ Received 2 doses

Against severe outcomes

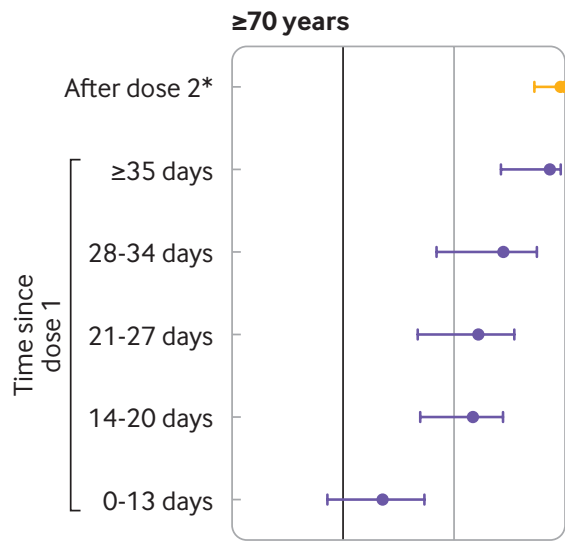

40-69 years

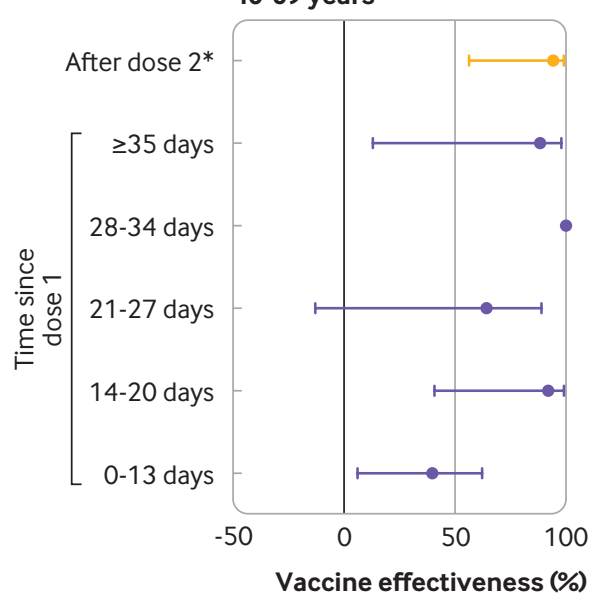

Fig 5 | Adjusted vaccine effectiveness estimates $\geq 14$ days after dose 1 and $\geq 0$ days after dose 2 of covid-19 mRNA vaccines (BNT162b2, mRNA-1273) against laboratory confirmed symptomatic SARS-CoV-2 infection and severe outcomes (hospital admission or death) by age group, between 14 December 2020 and 19 April 2021 in Ontario, Canada. Models were adjusted for age, sex, public health unit region, biweekly period of test, number of SARSCoV-2 tests in the three months before 14 December 2020 , presence of any comorbidity increasing the risk of severe covid-19, receipt of influenza vaccination in current or previous influenza season, and fifths of neighbourhood level household income, number of people living in each dwelling, proportion of people employed as non-health essential workers, and self-identified visible minority (unless adjusted variable was used for stratification). *For vaccine effectiveness estimates against symptomatic SARS-CoV- 2 infection, this interval was $\geq 7$ days after dose 2 . Against severe outcomes, vaccine effectiveness was evaluated for the entire period ( $\geq 0$ days) after receipt of the second dose owing to the small number of outcomes 
on the SARS-CoV-2 laboratory requisition form into the Ontario Laboratories Information System. Thus, the generalisability of our findings to the broader population is uncertain and we could not estimate vaccine effectiveness against asymptomatic infection. However, the per cent positivity of our study sample $(53270 / 324033=16.4 \%)$ did not differ much from that observed in Ontario during the study period $(281261 / 2171449=13.0 \%)$, and we would expect positivity to be higher for people with symptoms than those without symptoms. We also acknowledge that those with no information on symptoms recorded in the Ontario Laboratories Information System might have had symptoms at the time of testing, and those recorded as asymptomatic might have subsequently developed symptoms. In addition, covid-19 vaccination status might also be collected on the laboratory requisition form. This could bias the true vaccine effectiveness estimate, depending on whether symptoms were more likely to be documented on requisition forms for vaccinated people who ultimately test positive for SARS-CoV-2 (this would bias vaccine effectiveness towards the null) or less likely to be recorded (this would bias vaccine effectiveness away from the null). To minimise this selection bias, traditional test negative design studies collect vaccination status among all people with symptoms consistent with the pathogen under study. However, the congruence of our findings for fully vaccinated people with extant studies provides some reassurance that any underestimation or overestimation of vaccine effectiveness is likely to be small. Secondly, because the date of symptom onset is largely unavailable in the Ontario Laboratories Information System, and the Case and Contact Management system only has information on people who test positive, we used specimen collection date as the index date. This might have led to some vaccinated people being classified into an incorrect dose-to-index interval because symptom onset would have occurred several days before being tested. The impact of earlier intervals on vaccine effectiveness estimates if using the specimen collection date depends on the test results for vaccinated people (eg, vaccine effectiveness for earlier intervals would be overestimated if there were more vaccinated test positive cases and underestimated if there were more vaccinated test negative controls) or whether the lag between symptom onset and specimen collection dates resulted in misclassification of vaccinated people (false negatives), which would overestimate vaccine effectiveness for earlier intervals. Furthermore, we could not limit the study population to those tested within 10 days of symptom onset, a commonly used inclusion criterion for test negative studies. Prolonging the interval between symptom onset and testing increases the likelihood of false negative cases, which lowers vaccine effectiveness estimates. However, $89 \%$ of cases with dates for both symptom onset and specimen collection documented in the Case and Contact Management system (not the source of symptom data for this study) were tested within 10 days of symptom onset. Thirdly, our results might have been affected by outcome misclassification of severe outcomes due to unlinked case records and incomplete capture of severe outcomes in the Case and Contact Management system, and delays in identifying hospital admissions in the Discharge Abstract Database (which depends on people being discharged) and deaths in the Registered Persons Database. The direction of bias to vaccine effectiveness estimates depends on whether data completeness and lags are differential between vaccinated and unvaccinated test positive cases. For example, if vaccination status ascertained during the case management process influenced the degree of data collection (eg, if more complete among vaccinated cases), vaccine effectiveness would be biased towards the null, or if unvaccinated cases have a prolonged hospital admission because of more severe course of illness, their hospital record would not be available for analysis and vaccine effectiveness would be biased towards the null. Fourthly, some of our covariates might be subject to measurement error. We used frequency of previous SARS-CoV-2 tests as a proxy to identify those at higher risk of exposure (and increased likelihood to be targeted for early vaccination). However, we did not include point of care tests because they are incompletely captured in the Ontario Laboratories Information System. Furthermore, since access to testing is variable, we might not have adequately controlled for this situation. Finally, we might not have adequately accounted for confounding bias with the covariates that were available in the study databases, especially against hospital admissions and deaths.

\section{Conclusions}

Our findings suggest that older people and those with comorbidities might benefit from risk based recommendations to minimise delays before a second dose of mRNA covid-19 vaccine. However, increasing protection against severe outcomes-arguably the more important outcomes-with increasing time after a first dose provides support for delaying the second dose in settings with constraints on vaccine supply. Mathematical modelling could be done to show how, particularly for jurisdictions with limited vaccine supply, vaccines should be distributed to maximise the protection of populations (eg, the relative benefits of providing second doses earlier to older populations versus providing more first doses to younger populations who respond better to one dose and thereby leading to more rapid achievement of herd immunity by maximising coverage with one dose). Since vaccine effectiveness against symptomatic infection after one dose is only moderate, and among older adults appears to be modest even at 14-20 days, people need to be informed that besides the absence of benefit during the first two weeks (and likely longer for older adults) after one dose of a mRNA covid-19 vaccine, they should continue to adhere to recommended public health measures, such as wearing a mask, physical distancing, and avoidance of social gatherings. 
AUTHOR AFFILIATIONS

\section{${ }^{1}$ ICES, Toronto, ON, Canada}

${ }^{2}$ Dalla Lana School of Public Health, University of Toronto, Toronto, ON, Canada

${ }^{3}$ Public Health Ontario, ON, Canada

${ }^{4}$ Centre for Vaccine Preventable Diseases, University of Toronto, Toronto, ON, Canada

${ }^{5}$ School of Epidemiology and Public Health, University of Ottawa, ON, Canada

${ }^{6}$ Children's Hospital of Eastern Ontario Research Institute, Ottawa, ON, Canada

${ }^{7}$ Institute of Health Policy, Management, and Evaluation, University of Toronto, Toronto, ON, Canada

${ }^{8}$ Bruyère and Ottawa Hospital Research Institutes, Ottawa, ON, Canada

${ }^{9}$ Department of Medicine, University of Ottawa, Ottawa, ON, Canada

${ }^{10}$ Department of Laboratory Medicine and Pathobiology, University of Toronto, Toronto, ON, Canada

${ }^{11}$ Department of Epidemiology, Biostatistics, and Occupational Health, School of Population and Global Health, McGill University, Montreal, QC, Canada

${ }^{12}$ Vaccine and Drug Evaluation Centre, Department of Community Health Sciences, University of Manitoba, Winnipeg, MB, Canada

${ }^{13}$ Analytics and Performance Reporting Branch, Alberta Health, Edmonton, $A B$, Canada

${ }^{14}$ Division of Preventive Medicine, Faculty of Medicine and Dentistry, University of Alberta, Edmonton, AB, Canada

${ }^{15}$ School of Public Health, University of Alberta, Edmonton, AB, Canada

${ }^{16}$ Department of Community Health Sciences, Cumming School of Medicine, University of Calgary, Calgary, AB, Canada

${ }^{17}$ Faculty of Nursing, University of Alberta, Edmonton, AB, Canada

${ }^{18}$ British Columbia Centre for Disease Control, Vancouver, BC, Canada

${ }^{19}$ School of Population and Public Health, University of British Columbia, Vancouver, BC, Canada

${ }^{20}$ Women's College Hospital, Toronto, ON, Canada

${ }^{21}$ Leslie Dan Faculty of Pharmacy, University of Toronto, Toronto, ON, Canada

${ }^{22}$ Department of Family and Community Medicine, University of Toronto, Toronto, ON, Canada

${ }^{23}$ University Health Network, Toronto, ON, Canada

We thank Public Health Ontario for access to case level data from the Public Health Case and Contact Management system and covid-19 laboratory data, as well as assistance with data interpretation; the staff of Ontario's public health units who are responsible for covid-19 case and contact management and data collection within the Case and Contact Management system; IQVIA Solutions Canada for use of its Drug Information Database; and the Ontario residents without whom this research would be impossible.

Contributors: $\mathrm{HC}$ and JCK designed and oversaw the study. SH and HC obtained the data and conducted all analyses (dataset and variable creation and statistical modelling). BC contributed to data analyses and data preparation for the symptomatic dataset. SN, MES, HC, and ICK drafted the manuscript. All authors contributed to the analysis plan, interpreted the results, critically reviewed and edited the manuscript, approved the final version, and agreed to be accountable for all aspects of the work. JCK is the guarantor. The corresponding author attests that all listed authors meet authorship criteria and that no others meeting the criteria have been omitted.

Funding: This work was supported by the Canadian Immunization Research Network (CIRN) through a grant from the Public Health Agency of Canada and the Canadian Institutes of Health Research (CNF 151944); funding from the Public Health Agency of Canada, through the Vaccine Surveillance Reference group and the COVID-19 Immunity Task Force; and ICES, which is funded by an annual grant from Ontario's Ministry of Health $(\mathrm{MOH})$ and Ministry of Long term Care (MLTC); and the Ontario Health Data Platform (OHDP), a Province of Ontario initiative to support Ontario's ongoing response to covid-19 and its related impacts. JCK is supported by a clinician-scientist award from the University of Toronto Department of Family and Community Medicine. PCA is supported by a mid-career investigator award from the Heart and Stroke Foundation. The study sponsors did not participate in the design and conduct of the study; collection, management, analysis, and interpretation of the data; preparation, review, or approval of the manuscript; or the decision to submit the manuscript for publication.

Parts of this material are based on data and/or information compiled and provided by the Canadian Institute for Health Information $(\mathrm{CIHI})$ and by Cancer Care Ontario (CCO). However, the analyses, conclusions, opinions, and statement expressed herein are solely those of the authors and do not reflect those of the funding or data sources; no endorsement by ICES, MOH, MLTC, OHDP, its partners, the Province of Ontario, $\mathrm{ClHI}$, or CCO is intended or should be inferred.

Competing interests: All authors have completed the ICMJE uniform disclosure form at www.icmje.org/coi_disclosure.pdf and declare: support from the Public Health Agency of Canada, the Canadian Institutes of Health Research, and Ontario's Ministry of Health and Ministry of Long term Care for the submitted work. KW is chief executive officer of CANImmunize and serves on the data safety board for the Medicago covid-19 vaccine trial. SMM has received unrestricted research grants from Merck, GlaxoSmithKline, Sanofi Pasteur, Pfizer, and Roche-Assurex for unrelated studies. SMM has received fees as an advisory board member for GlaxoSmithKline, Merck, Pfizer, Sanofi Pasteur, and Seqirus. CHR has received an unrestricted research grant from Pfizer for an unrelated study.

Ethical approval: Projects that use data collected by ICES under section 45 of Ontario's Personal Health Information Protection Act (PHIPA), and use no other data, are exempt from research ethics board review. The use of the data in this project is authorised under section 45 and approved by ICES' Privacy and Legal Office. ICES is a prescribed entity under PHIPA. Section 45 of PHIPA authorises ICES to collect personal health information, without consent, for the purpose of analysis or compiling statistical information with respect to the management of, evaluation or monitoring of, the allocation of resources to or planning for all or part of the health system.

Data sharing: The study dataset is held securely in coded form at ICES. While legal data sharing agreements between ICES and data providers (eg, healthcare organisations and government) prohibit ICES from making the dataset publicly available, access might be granted to those who meet prespecified criteria for confidential access, available at www.ices.on.ca/DAS (email das@ices.on.ca).The full dataset creation plan and underlying analytic code are available from the authors upon request, understanding that the computer programs might rely upon coding templates or macros that are unique to ICES and are therefore either inaccessible or require modification.

The corresponding author (the manuscript's guarantor) affirms that the manuscript is an honest, accurate, and transparent account of the study being reported; that no important aspects of the study have been omitted; and that any discrepancies from the study as planned have been explained.

Dissemination to participants and related patient and public communities: As the personal identifying information of participants have been removed from the study dataset, it is not possible to send the results of this study to participants. However, the results from this manuscript have been made publicly available through a preprint server and will be further disseminated by ICES through social media channels and news media.

Provenance and peer review: Not commissioned; externally peer reviewed.

This is an Open Access article distributed in accordance with the terms of the Creative Commons Attribution (CC BY 4.0) license, which permits others to distribute, remix, adapt and build upon this work, for commercial use, provided the original work is properly cited. See: http://creativecommons.org/licenses/by/4.0/.

1 Patel MM, Jackson ML, Ferdinands J. Postlicensure Evaluation of COVID-19 Vaccines. JAMA 2020;324:1939-40. doi:10.1001/ jama.2020.19328

2 National Advisory Committee on Immunization. COVID-19 vaccine: Guidance on the prioritization of initial doses - Canada.ca. 2021. https:// www.canada.ca/en/public-health/services/immunization/nationaladvisory-committee-on-immunization-naci/guidance-prioritizationinitial-doses-covid-19-vaccines.html (accessed 2 May 2021).

3 Jackson ML, Nelson JC. The test-negative design for estimating influenza vaccine effectiveness. Vaccine 2013;31:2165-8. doi:10.1016/j.vaccine.2013.02.053

4 Sullivan SG, Tchetgen Tchetgen EJ, Cowling BJ. Theoretical Basis of the Test-Negative Study Design for Assessment of Influenza Vaccine Effectiveness. Am J Epidemiol 2016;184:345-53. doi:10.1093/aje/ kww064 
5 Watson J, Whiting PF, Brush JE. Interpreting a covid-19 test result. BM/ 2020;369:m1808. doi:10.1136/bmj.m1808

6 Updates to PHO's Variants of Concern Laboratory Testing | Public Health Ontario. 2021. https://www.publichealthontario.ca/en/about/ blog/2021/lab-voc-testing-updates (accessed 3 May 2021)

7 Ontario's COVID-19 vaccination plan | COVID-19 (coronavirus) in Ontario. 2021. https://covid-19.ontario.ca/ontarios-covid-19vaccination-plan\#our-three-phased-vaccination-plan (accessed 3 May 2021).

8 Vaccine Clinical Advisory Group (VCAG) Recommendations on Exceptions to Extended Dose Intervals for COVID-19 vaccines. Ontario Ministry of Health 2021. https://www.health.gov.on.ca/en/ pro/programs/publichealth/coronavirus/docs/vaccine/COVID_19_ medical_exceptions_vaccine_dose_intervals.pdf (accessed 3 May 2021).

9 Extension of the Second Dose. Ontario Ministry of Health 2021. https://www.health.gov.on.ca/en/pro/programs/publichealth/ coronavirus/docs/vaccine/COVID_19_vaccine_dose_intervals.pdf (accessed 3 May 2021).

10 ICES COVID-19 Dashboard. 2021. https://www.ices.on.ca/DAS/ AHRQ/COVID-19-Dashboard (accessed 3 May 2021).

11 Underlying medical conditions associated with high risk for severe COVID-19: Information for healthcare providers. 2021 https://www.cdc.gov/coronavirus/2019-ncov/hcp/clinical-care/ underlyingconditions.html (accessed 3 May 2021).

12 Kwong JC, Buchan SA, Chung H, et al. Can routinely collected laboratory and health administrative data be used to assess influenza vaccine effectiveness? Assessing the validity of the Flu and Other Respiratory Viruses Research (FOREVER) Cohort. Vaccine 2019;37:4392-400. doi:10.1016/j.vaccine.2019.06.011

13 Dictionary, Census of Population, 2016 - Dissemination area (DA). Statistics Canada/Statistique Canada 2016. https://www12. statcan.gc.ca/census-recensement/2016/ref/dict/geo021-eng.cfm (accessed 24 Jul 2021).

14 Sundaram ME, Calzavara A, Mishra S, et al. Individual and social determinants of SARS-CoV-2 testing and positivity in Ontario, Canada: a population-wide study. CMAI 2021;193:E723-34. doi:10.1503/ cmaj.202608

15 Evaluation of COVID-19 vaccine effectiveness. 2021. https:// www.who.int/publications/i/item/WHO-2019-nCoV-vaccine effectiveness-measurement-2021.1 (accessed 24 Jul 2021).

16 Dagan N, Barda N, Kepten E, et al. BNT162b2 mRNA Covid-19 Vaccine in a Nationwide Mass Vaccination Setting. N Engl J Med 2021;384:1412-23. doi:10.1056/NEJMoa2101765

17 Hitchings MDT, Lewnard JA, Dean NE, et al. Use of recently vaccinated individuals to detect bias in test-negative case-control studies of COVID-19 vaccine effectiveness.medRxiv 2021

18 Mason T, Whitston M, Hodgson J, et al. Effects of BNT162b2 mRNA vaccine on Covid-19 infection and hospitalisation among olde people: matched case control study for England.medRxiv 2021

19 Haas EJ, Angulo FJ, McLaughlin JM, et al. Impact and effectiveness of mRNA BNT162b2 vaccine against SARS-CoV-2 infections and COVID-19 cases, hospitalisations, and deaths following a nationwide vaccination campaign in Israel: an observational study using national surveillance data. Lancet 2021;397:1819-29. doi:10.1016/S01406736(21)00947-8

20 Thompson MG, Burgess JL, Naleway AL, et al. Interim Estimates of Vaccine Effectiveness of BNT162b2 and mRNA-1273 COVID-19 Vaccines in Preventing SARS-CoV-2 Infection Among Health Care Personnel, First Responders, and Other Essential and Frontline Workers - Eight U.S. Locations, December 2020-March 2021. MMWR Morb Mortal Wkly Rep 2021;70:495-500. doi:10.15585/mmwr. mm7013e3

21 Angel Y, Spitzer A, Henig O, et al. Association Between Vaccination With BNT162b2 and Incidence of Symptomatic and Asymptomatic SARS-CoV-2 Infections Among Health Care Workers. JAMA 2021;325:2457-65. doi:10.1001/jama.2021.7152

22 Tang L, Hijano DR, Gaur AH, et al. Asymptomatic and Symptomatic SARS-CoV-2 Infections After BNT162b2 Vaccination in a Routinely Screened Workforce. JAMA 2021;325:2500-2. doi:10.1001/ jama.2021.6564

23 Tenforde MW, Olson SM, Self WH, et al, IVY Network, HAIVEN Investigators. Effectiveness of Pfizer-BioNTech and Moderna Vaccines Against COVID-19 Among Hospitalized Adults Aged $\geq 65$ Years - United States, January-March 2021. MMWR Morb Mortal Wkly Rep 2021;70:674-9. doi:10.15585/mmwr.mm7018e1
24 Björk J, Inghammar M, Moghaddassi M, et al. Effectiveness of the BNT162b2 vaccine in preventing COVID-19 in the working age population - first results from a cohort study in Southern Sweden. medRxiv 2021.

25 Lopez Bernal J, Andrews N, Gower C, et al. Effectiveness of the PfizerBioNTech and Oxford-AstraZeneca vaccines on covid-19 related symptoms, hospital admissions, and mortality in older adults in England: test negative case-control study. BMJ 2021;373:n1088. doi:10.1136/bmj.n1088

26 Pawlowski C, Lenehan P, Puranik A, et al. FDA-authorized mRNA COVID-19 vaccines are effective per real-world evidence synthesized across a multi-state health system. Med (N Y) 2021. doi:10.1016/J. MEDJ.2021.06.007

27 Aran D. Estimating real-world COVID-19 vaccine effectiveness in Israel using aggregated counts.medRxiv 2021

28 Yelin I, Katz R, Herzel E, et al. Associations of the BNT162 b2 COVID-19 vaccine effectiveness with patient age and comorbidities. medRxiv 2021.

29 Vasileiou E, Simpson CR, Shi T, et al. Interim findings from firstdose mass COVID-19 vaccination roll-out and COVID-19 hospital admissions in Scotland: a national prospective cohort study. Lancet 2021;397:1646-57. doi:10.1016/S0140-6736(21)00677-2

30 Andrejko KL, Pry J, Myers JF, et al. Prevention of COVID-19 by mRNA-based vaccines within the general population of California. medRxiv 2021.

31 Hall VJ, Foulkes S, Saei A, et al, SIREN Study Group. COVID-19 vaccine coverage in health-care workers in England and effectiveness of BNT162b2 mRNA vaccine against infection (SIREN): a prospective, multicentre, cohort study. Lancet 2021;397:1725-35. doi:10.1016 S0140-6736(21)00790-X

32 Chodick G, Tene L, Patalon T, et al. The effectiveness of the first dose of BNT162b2 vaccine in reducing SARS-CoV-2 infection 13-24 days after immunization: real-world evidence.medRxiv 2021;2021.01.27.21250612. doi:10.1101/2021.01.27.21250612

33 Skowronski DM, De Serres G. Safety and Efficacy of the BNT162b2 mRNA Covid-19 Vaccine. N Engl J Med 2021;384:1576-7. doi:10.1056/NEJMc2036242.

34 Public Health England. Annex A: Report to JCVI on estimated efficacy of a single dose of Pfizer BioNTech (BNT162b2 mRNA) vaccine and of a single dose of ChAdOx1 vaccine (AZD1222). PHE 2020

35 Hyams C, Marlow R, Maseko Z, et al. Effectiveness of BNT162b2 and ChAdOx1 nCoV-19 COVID-19 vaccination at preventing hospitalisations in people aged at least 80 years: a test-negative, case-control study. Lancet Infect Dis 2021;S1473-3099(21)003303. doi:10.1016/S1473-3099(21)00330-3

36 COVID-19 Alberta statistics. https://www.alberta.ca/stats/covid-19alberta-statistics.htm\#vaccine-outcomes (accessed 24 Jul 2021).

37 Swift MD, Breeher LE, Tande AJ, et al. Effectiveness of mRNA COVID-19 vaccines against SARS-CoV-2 infection in a cohort of healthcare personnel. Clin Infect Dis 2021;26:ciab361. doi:10.1093/cid/ciab361

38 COVID-19 vaccine surveillance report - week 26. Public Health England 2021. https://assets.publishing.service.gov.uk/government/ uploads/system/uploads/attachment_data/file/998411/Vaccine_ surveillance_report_-_week_26.pdf (accessed 24 Jul 2021).

39 Butt AA, Omer SB, Yan P, Shaikh OS, Mayr FB. SARS-CoV-2 Vaccine Effectiveness in a High-Risk National Population in a Real-World Setting. Ann Intern Med 2021. doi:10.7326/M21-1577

40 Thompson MG, Burgess JL, Naleway AL, et al. Prevention and Attenuation of Covid-19 with the BNT162b2 and mRNA-1273 Vaccines. N Engl J Med 2021;385:320-9. doi:10.1056/NEJMoa2107058

41 Chemaitelly H, Yassine HM, Benslimane FM, et al. mRNA-1273 COVID-19 vaccine effectiveness against the B.1.1.7 and B.1.351 variants and severe COVID-19 disease in Qatar. Nat Med 2021. doi:10.1038/S41591-021-01446-Y

42 Hunter PR, Brainard J. Estimating the effectiveness of the Pfizer COVID-19 BNT162b2 vaccine after a single dose. A reanalysis of a study of 'real-world' vaccination outcomes from Israel.medRxiv 2021

43 Goldfarb JL, Kreps S, Brownstein JS, Kriner DL. Beyond the First Dose - Covid-19 Vaccine Follow-through and Continued Protective Measures. N Engl J Med 2021;385:101-3. doi:10.1056/ NEJMP2104527

Supplementary information: additional methods, eTables 1-8, and eFigures 1 and 2 